\title{
A supermartingale argument for characterizing the Functional Hill process weak law for small parameters
}

\author{
Dr. A. M. Fall* \\ G. S. $\mathrm{LO}^{\dagger}$ \\ A. Adekpedjou ${ }^{\ddagger}$ \\ C. H. Ndiaye ${ }^{\S}$
}

November 13, 2018

\begin{abstract}
The paper deals with the asymptotic laws of functionals of standard exponential random variables. These classes of statistics are closely related to estimators of the extreme value index when the underlying distribution function is in the Weibull domain of attraction. We use techniques based on martingales theory to describe the non Gaussian asymptotic distribution of the aforementioned statistics. We provide results of a simulation study as well as statistical tests that may be of interest with the proposed results
\end{abstract}

Keywords: Supermartingale; Functional Hill process; Extreme value theory

*A. M. Fall (adjambarkafall@gmail.com) is a seasonal professor, University Gaston Berger (UGB), Saint Louis, Senegal;

${ }^{\dagger}$ G. S. Lo (gane-samb.lo@ugb.edu.sn) is a Professor, UGB, Saint Louis, Senegal; and African University of Sciences and technolgy (AUST), Abuja, Nigeria; and Associated to LSTA, Pierre et Marie Curie University, Paris, France;

${ }_{\ddagger}^{\ddagger}$ A. Adekpedjou (akima@mst.edu) is Associate Professor, Missouri S\&T, Rolla, MO, 65409-USA

${ }^{\S}$ C. H. Ndiaye (ham111266@yahoo.fr) LMA, UCAD, Dakar, Senegal 


\section{Introduction}

Of interest in this manuscript are the asymptotic properties of functional stochastic processes based on extreme values of independent and identically distributed (iid) random variables $(r v) X_{1}, X_{2}, \ldots$, wich are defined on the same probability space $(\Omega, \mathcal{P}, \mathbb{P})$, whose common distribution function is $F$.

Let $X_{1, n} \leq \cdots \leq X_{n, n}$ be the associated order statistics and $k(n)$ a sequence of integers satisfying $1 \leq k(n)<n$.

Since we are only interested by the upper tail of $F$, we may and do suppose that $X_{i}>0$. Accordingly, we will have to use the log transform $Y=\log X$ to get iid rv's $Y_{i}=\log X_{i}, i \geq 1$, with common distribution function $(d f) G(x)=F\left(e^{x}\right)$ such that their order statistics satisfy $\left\{Y_{i, n}, 1 \leq i \leq n\right\}=\left\{\log X_{i, n}, 1 \leq i \leq n\right\}$ for each $n \geq 1$.

Let $f(j)$ be a real and increasing function of the integer $j$ such that $f(0)=0$. The following empirical process, hereon called the functional Hill process,

$$
T_{n}(f)=\sum_{j=1}^{k(n)} f(j)\left(\log X_{n-j+1, n}-\log X_{n-j, n}\right)
$$

was introduced by Deme et al. (2012). A generalization of the Diop et al. statistic given by

$$
\sum_{j=1}^{k(n)} j^{\tau}\left(\log X_{n-j+1, n}-\log X_{n-j, n}\right) / k^{\tau}(n),
$$

for $f(j)=j^{\tau}, \tau>0, j \geq 1$ and $f(0)=0$ is obtained when (1) is divided by $f(k)$ (cf. Diop and Lo (1994) and Diop and Lo (2009)). The statistic (2) is a direct generalization of the classical Hill's estimator obtained for $\tau=1$. If $K$ is some Kernel function (cf. Goegebeur et al. (2010) and Groeneboom et 
al. (2003)), these statistics are closely related to the Kernel-type estimators like the one due to Csörgő et al. (1985) and given by

$$
\left\{\sum_{j=1}^{k(n)} j K(j / k(n))\left(\log X_{n-j+1, n}-\log X_{n-j, n}\right) / k(n)\right\} /\left\{\sum_{j=1}^{k(n)} K(j / k(n))\right\} .
$$

The statistics (2) and (3) are generalizations of the Hill estimator corresponding to $K=1$ in (3) and $\tau=1$ in (2) respectively, the later playing a crucial role in Extreme Value Theory (EVT). EVT is a theory whose main purpose pertains to the derivation of the asymptotic distribution of the maximum order statistics for a random sample $X_{1}, \ldots, X_{n}$, usually denoted by $X_{n, n}$. The distribution of $X_{n, n}$ has applications in many areas such as actuarial science in the determination of the distributional properties of the largest claim, or in survival analysis to determine the asymptotic properties of the largest failure time of items subject to failure or censored data to name a few.

One says that the underlying distribution function $F$ of the $X_{i}$ 's is attracted to another distribution function $H$ if for some sequences $\left(a_{n}>0\right)_{n \geq 1}$ and $\left(b_{n}\right)_{n \geq 1}$, and for any real point of continuity $x$ of $H$, the following holds

$$
\lim _{n \rightarrow \infty} P\left(\frac{X_{n, n}-b_{n}}{a_{n}} \leq x\right)=\lim _{n \rightarrow \infty} F^{n}\left(a_{n} x+b_{n}\right)=H(x)
$$

If $H$ is non-degenerated, then it can be parameterized as $G_{\gamma}(x)=\exp [-(1+$ $\left.\gamma x)^{-1 / \gamma}\right] I\{1+\gamma x>0\}$ with $\gamma \neq 0$, where $I\{\cdot\}$ denotes the indicator function and $G_{0}(x)=\exp (-\exp (-x))$. Here, the function $G_{\gamma}(x)$ is called the Generalized Extreme Value (GEV) distribution. If property (4) holds, one says that $F$ is in the domain of attraction of $H$, and we write $F \in D(H)$. The reader is referred to de Haan and Ferreira (2006), Resnick (1987), Galambos (1985), Beirlant et al. (2004), and Embrechts et al. (1997) for a modern account of EVT. Although the parameter $\gamma$ in the GEV distribution is con- 
tinuous, the three cases $\gamma<0, \gamma=0$ and $\gamma>0$, named after the Weibull, Gumbel and Frechet domains of attraction respectively have different distributional properties. However, in all three cases, the Hill statistic is used to estimate the extreme value index corresponding to $\tau=1$ in (2).

(1) In the case of $\gamma \geq 0$ for instance, (2) converges in probability to $\gamma$ as $n \rightarrow \infty$ and $k / n \rightarrow 0$.

(2), For $\gamma<0$, the upper endpoint of $G(x)=F\left(e^{x}\right)$ defined by $y_{0}=$ $\sup \{x \in \Re, G(x)<1\}$ is finite, and it is related to the upper endpoint of $F$, $x_{0}=\sup \{x \in \Re, F(x)<1\}$ by $y_{0}=\log x_{0}$. If $G^{-1}$ stands for the generalized inverse function of $G$, then (2), when normalized by $y_{0}-G^{-1}(1-k(n) / n)$, converges to $(1-\gamma)^{-1}$ as $n \rightarrow \infty$ and $k(n) / n \rightarrow 0$.

The Diop and Lo (2009) generalization of the Hill's estimator which corresponds to (2) was introduced in Diop and Lo (1994) and further studied in Diop and Lo (2009) where its asymptotic normality was established for any $\gamma$, but only for $\tau>1 / 2$. It is not possible to obtain the asymptotic distribution of the Diop and Lo estimator for $\tau \leq 1 / 2$ via Hungarian Gaussian approximation that was utilized then. Recently, the statistic obtained by dividing the expression in (11) by $f(k)$, which generalizes (2) for $f(j)=j^{\tau}$, has been extensively studied for the Frechet and Gumbel distributions by Deme et al.(2012). When dealing with the family of functions $f(j)=j^{\tau}$, parameterized by $\tau>0$, we will use the notation $f_{\tau}(j)=j^{\tau}$. In the aforementioned paper, Deme et al. (2012) proved that (1) has a limiting Gaussian process when $A(2, f)=: \sum_{j=1}^{+\infty} f(j)^{2} / j^{2}=\infty$ and

$$
B_{n}(f)=: \frac{\max \{f(j) / j, 1 \leq j \leq k\}}{\sqrt{\sum_{j=1}^{k} f(j)^{2} / j^{2}}}
$$

converges to 0 as $n \rightarrow \infty$. However, when $A(2, f)<+\infty$, (1) has a non 
Gaussian limiting process. Applying these results for the class of functions $f_{\tau}(\cdot)$, we get that for $\gamma>0$, the asymptotic law of (2) is Gaussian for $\tau \geq 1 / 2$ and non-Gaussian for $0<\tau<1 / 2$. Coupling those observations with the results of Diop and Lo (2009), we can easily conclude that the limiting distribution of (2) is known except for the Weibull domain and for $0<\tau \leq 1 / 2$.

The problem of finding the asymptotic distribution of $(2)$ in the Weibull domain when $0<\tau \leq 1 / 2$ has not been investigated. In this manuscript, we take a supermartingale approach to obtain the limiting distribution of the generalized Hill statistics given in (2) in the said domain. The supermartingale approach was motivated by the fact that under condition $A(2, f)<\infty$, (1), when properly centered and scaled can be written as a sum of dependent random variables. With that approach and a complete characterization of the process

$$
\sum_{j=1}^{k-1}(f(j)-f(j-1))\left[\exp \left(-\gamma \sum_{h=j}^{k-1} E_{h} / h\right)-E \exp \left(-\gamma \sum_{h=j}^{k-1} E_{h} / h\right)\right]
$$

we were able to find the asymptotic distribution of (1) and (2) for $0<\tau<$ $1 / 2$.

The manuscript will proceed as follow. In Section 2, we study a special process based on a sequence of iid unit exponential rvs whose limiting distribution will be found via martingale techniques. Section 3 is devoted to the application of the results of section 2 to our problem. The results of a simulation study are presented in section 4 along with some results pertaining to statistical tests in the extreme value domains. The manuscript will conclude with a discussion section and an appendix. 


\section{A supermartingale tool}

Let $E_{1}, E_{2}, \ldots$ be iid unit exponentially distributed rvs. Let $k \geq 1$. Let also $\gamma>0$ be positive number that is constant throughout this paper. Define the increments of $f$ by $\Delta f(j)=f(j)-f(j-1)$ for $j=1,2, \ldots$. Let also the sequence $W_{k}(f)$ be given by the sequence

$$
W_{k}(f)=\sum_{j=1}^{k} \Delta f(j)\left(\exp \left(-\gamma \sum_{h=j}^{k} E_{h} / h\right)-E \exp \left(-\gamma \sum_{h=j}^{k} E_{h} / h\right)\right)
$$

for $k \in \aleph$. Consider the filtration $\mathcal{F}_{k}=\sigma\left(E_{1}, \ldots, E_{k}\right), k \geq 1$ and observe that the sequence $\left(W_{k}\right)_{k \geq 1}$ is adapted to $\left(\mathcal{F}_{k}\right)_{k \geq 1}$. We first introduce some intermediate results that will prove to be crucial for the proof of our main results. The next two ones pertain to the martingale property of $W_{k}(f)$ and its limiting distribution.

Theorem 1 The sequence $\left(W_{k}(f)\right)_{k \geq 1}$ is a supermatingale with respect to $\mathcal{F}_{k}$. Furthermore, it converges almost-surely (a.s) to a random variable $W_{\infty}(f)$ with finite expectation whenever

$$
\limsup _{k \rightarrow+\infty} k^{-\gamma} \sum_{j=L}^{k-1} \Delta f(j) j^{\gamma-1 / 2}<+\infty
$$

holds.

Corollary 1 For $f(j)=f_{\tau}(j)=j^{\tau}, 0<\tau<1 / 2, W_{k}\left(f_{\tau}\right)$ converges almost surely to a finite expectation random variable $W_{\infty}^{*}(\tau)$.

Remark Actually, the random variable $W_{\infty}^{*}(\tau)$ given in Corollary 1 is exactly $W_{\infty}\left(f_{\tau}\right)$ of Theorem 1, where the function $f_{\tau}$ is defined in Corollary 1 . To make the notations simple, we will simply write $W_{\infty}^{*}(\tau)=W_{\infty}(\tau)$, when the argument is a positive number. 
Proof of theorem 1: Let

$$
S_{j, k}=\exp \left(-\gamma \sum_{h=j}^{k} E_{h} / h\right)
$$

and $V_{k}=\exp \left(-\gamma E_{k} / k\right)$ for $k \geq 1$. We have for $k \geq 1$

$W_{k+1}=\sum_{j=1}^{k} \Delta f(j)\left(S_{j, k} V_{k+1}-\mathrm{E} \exp \left(S_{j, k} V_{k+1}\right)\right)+\Delta f(k+1)\left(V_{k+1}-\mathrm{E} \exp \left(V_{k+1}\right)\right)$

Observe that: (1) $V_{k+1}$ and $S_{j, k}$ are independent for $1 \leq j \leq k,(2) V_{k+1}$ is independent of $\mathcal{F}_{k}$ and (3) an application of moment generating function for unit exponential distribution yields $E\left(V_{k+1}\right)=(1+\gamma /(k+1))^{-1}$.

From now on, the notation $\ell(k, \gamma)=(1+\gamma /(k+1))^{-1}$ is used to make the formulas simple. The three previous observations, when combined together, lead to $\mathrm{E}\left(\left(V_{k+1}-\mathrm{E} \exp \left(V_{k+1}\right)\right) \mid \mathcal{F}_{k}\right)=0$ and

$$
\mathrm{E}\left(W_{k+1} \mid \mathcal{F}_{k}\right)=\mathrm{E}\left(V_{k+1}\right) \sum_{j=1}^{k+1} \Delta f(j)\left(S_{j, k}-\mathrm{E} \exp \left(S_{j, k}\right)\right)=\ell(k+1, \gamma) W_{k}
$$

Since the function $\ell(k, \gamma)$ is increasing in $k$, we obtain

$$
\mathrm{E}\left(\frac{W_{k+1}}{\ell(k+1, \gamma)} \mid \mathcal{F}_{k}\right)=\frac{W_{k}}{\ell(k, \gamma)} \times \frac{\ell(k, \gamma)}{\ell(k+1, \gamma)} \leq \frac{W_{k}}{\ell(k, \gamma)}
$$

Therefore, $W_{k} / \ell(k, \gamma)$ is a supermartingale. A sufficient condition of a.s. convergence of $W_{k} / \ell(k, \gamma)$, as $k \rightarrow+\infty$ ( $\gamma$ is fixed) to a finite expectation $r v$ is

$$
\limsup _{k \rightarrow+\infty} \mathrm{E}\left(\left|W_{k}\right|\right)<\infty
$$

since $\ell(k, \gamma)$ converges to unity when $k \rightarrow \infty, \gamma$ being fixed.. Next, by denoting $s_{j, k}=E\left(S_{j, k}\right), 1 \leq j \leq k, k \geq 1$ and by using Cauchy-Schwarz and 
Minkowski inequalities, we have

$$
\begin{gathered}
E\left|W_{k}\right| \leq\left(\mathrm{E}\left(W_{k}^{2}\right)\right)^{1 / 2}=\left\|W_{k}\right\|_{2} \leq \sum_{j=1}^{k}\left\|\Delta f(j)\left(S_{j, k}-s_{j, k}\right)\right\|_{2} \\
\leq \sum_{j=1}^{k-1} \Delta f(j)\left(\operatorname{Var}\left(S_{j, k}\right)\right)^{1 / 2} .
\end{gathered}
$$

In the appendix, we provide moments computations of the $S_{j, k}$ 's, especially concerning their expectations, variances and covariances. These computations are based on integral calculations given in subsection 6.2. By Formula (19), $\operatorname{Var}\left(S_{j, k}\right)$ is bounded by unity for any $1 \leq j \leq k$. Combining this with (27) and fixing $\varepsilon$ such that $0<\varepsilon \leq 1$, we get for $L$ large enough and $k-1>L$

$$
\begin{aligned}
\mathrm{E}\left|W_{k}\right| \leq & \sum_{j=1}^{L} \Delta f(j)\left(\operatorname{Var}\left(S_{j, k}\right)\right)^{1 / 2} \\
& +\frac{(2(1+\varepsilon)(\varepsilon+1 / 2))^{1 / 2} \gamma}{k^{\gamma}} \sum_{j=L+1}^{k-1} \Delta f(j) j^{\gamma-1 / 2} \\
\leq & \sum_{j=1}^{L} \Delta f(j)+\frac{4 \gamma}{k^{\gamma}} \sum_{j=L+1}^{k-1} \Delta f(j) j^{\gamma-1 / 2}
\end{aligned}
$$

Since the first term in the right hand side of $(8)$ is bounded for a fixed $L$, we see that the supremum limit of $\mathrm{E}\left|W_{k}\right|$ is finite whenever (K1) holds. This proves the theorem.

Proof of Corollary: Observe that for large values of $j, \Delta f(j) \sim \tau j^{\tau-1}$ and Condition (K1) is equivalent to the boundedness of

$$
k^{-\gamma} \sum_{j=L}^{k-1} j^{\tau+\gamma-3 / 2}
$$


Now, let $0<\tau \leq 1 / 2$ and consider the four possible cases: (i) $\tau+\gamma-3 / 2=$ -1 ; (ii) $\tau+\gamma-3 / 2<-1$; (iii) $\tau+\gamma-3 / 2=0$ and (iv) $\tau+\gamma-3 / 2>0$. Using (31), we get that (9) is less than $k^{-\gamma}(\log (k-1)-\log L)+(k-1)^{-1}$ for (i) and is less than $k^{-\gamma}\left((k-1)^{\gamma+(\tau-1 / 2)}-L^{\gamma+(\tau-1 / 2)}\right) /(\gamma+\tau-1 / 2)+(k-1)^{\gamma+(\tau-3 / 2)}$ for (ii). In the third case, $\sqrt{9}$ is exactly $k^{-\gamma}(k-1-L)=\left(k^{(\tau-1 / 2)}-(1+\right.$ $L) k^{-(3 / 2-\tau)}$. Finally, applying 32 to the last case, $(9)$ is found to be less than

$$
k^{-\gamma}\left((k-1)^{\gamma+(\tau-1 / 2)}-L^{\gamma+(\tau-1 / 2)}\right) /(\gamma+\tau-1 / 2)+L^{\gamma+(\tau-3 / 2)} .
$$

In all these four cases, we get that $\limsup _{k \rightarrow+\infty} k^{-\gamma} \sum_{j=L}^{k-1} j^{\tau+\gamma-3 / 2}<\infty$, and the statement in the corollary is proven.

\section{Main Results}

Let us give a brief statement about the problem of interest.

To make the notation compact, we set $k(n) \equiv k$. Recall that $G \in D\left(G_{-\gamma}\right)$ if and only if $F \in D\left(G_{-\gamma}\right)$. As mentioned earlier, this manuscript pertains to the properties of the leading part of (1) when $F \in D\left(G_{-\gamma}\right)$ and $0<\gamma<1 / 2$. We begin with the special case of functions $F \in D\left(G_{-\gamma}\right)$, that is

$$
y_{0}-G^{-1}(1-u)=u^{\gamma} I\{u \in[0,1]\}
$$

where $y_{0}$ is the upper endpoint of $G$. We use here the index $-\gamma<0$ instead of $\gamma<0$, hence condition 10 still holds. The next theorem characterizes the asymptotic distribution of $T_{n}(f)$ when Condition (K1) is satisfied.

Theorem 2 Let $X_{1}, X_{2}, \ldots$ be a sequence of iid rv's with common df $F$ such 
that (10) holds for $G(x)=F\left(e^{x}\right)$. Let $f(j)$ be an increasing function of $j \geq 0$, with $f(0)=0$ such that (K1) holds. For any $1 \leq k \leq n$, let

$$
A_{k, n}(f)=: f(k-1)-\sum_{j=1}^{k-1}(f(j)-f(j-1)) \exp \left(-\sum_{h=j}^{k-1} \log (1+\gamma / h)\right) .
$$

Then

$$
W_{k-1, n}^{*}(f):=A_{k, n}(f)-\frac{T_{n}(f)}{y_{0}-Y_{n-k+1, n}}
$$

converges in distribution to the finite expectation random variable $W_{\infty}(f)$ defined in Theorem 1. Furthermore, if $f(j)=f_{\tau}(j)=j^{\tau}$ for $0<\tau \leq 1 / 2$, then $W_{k-1, n}^{*}\left(f_{\tau}\right)$ converges in distribution to $W_{\infty}\left(f_{\tau}\right)=W_{\infty}^{*}(\tau)$ defined in Corollary 1 .

Proof of Theorem 2; The proof uses the classical representation of the $Y_{j}=\log X_{j}$ associated with the $d f G(x)=F\left(e^{x}\right)$ through a sequence of independent standard uniform rv's $U_{1}, U_{2}, \ldots$, that is

$$
\left\{Y_{j}, j \geq 1\right\}={ }_{d}\left\{G^{-1}\left(1-U_{j}\right), j \geq 1\right\}
$$

and then

$$
\left\{\left\{Y_{n-j+1, n}, 1 \leq j \leq n\right\}, n \geq 1\right\}={ }_{d}\left\{\left\{G^{-1}\left(1-U_{j, n}\right), 1 \leq j \leq n\right\}, n \geq 1\right\}
$$


This gives

$$
\begin{aligned}
\frac{T_{n}(f)}{y_{0}-Y_{n-k+1, n}} & =\sum_{j=1}^{k} f(j) \frac{\log X_{n-j+1, n}-\log X_{n-j, n}}{y_{0}-Y_{n-k+1, n}} \\
& =\sum_{j=1}^{k} f(j) \frac{\left(y_{0}-\log X_{n-j, n}\right)-\left(y_{0}-\log X_{n-j+1, n}\right)}{y_{0}-Y_{n-k+1, n}} \\
& ={ }_{d} \sum_{j=1}^{j=k} f(j)\left(\left(U_{j+1, n} / U_{k, n}\right)^{\gamma}-\left(U_{j, n} / U_{k, n}\right)^{\gamma}\right) .
\end{aligned}
$$

We have for $1 \leq j \leq k-1$

$$
\begin{aligned}
{\left[\frac{U_{j, n}}{U_{k, n}}\right]^{\gamma} } & =\prod_{h=j}^{k-1}\left[\frac{U_{h, n}}{U_{h+1, n}}\right]^{\gamma} \\
& =\exp \left(-\gamma \sum_{h=j}^{k-1} \frac{1}{h} \log \left(\frac{U_{h+1, n}}{U_{h, n}}\right)^{h}\right) \\
& \equiv \exp \left(-\gamma \sum_{h=j}^{k-1} E_{h}^{(n)} / h\right) .
\end{aligned}
$$

By the Malmquist representation (see proposition 31 in [14], p. 112 or

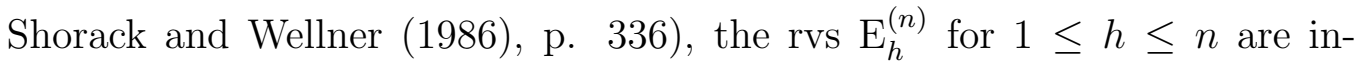
dependent unit exponential. From that observation, it follows that

$$
\frac{T_{n}(f)}{y_{0}-Y_{n-k+1, n}}=\sum_{j=1}^{k} f(j)\left[\exp \left(-\gamma \sum_{h=j+1}^{k-1} \mathrm{E}_{h}^{(n)} / h\right)-\exp \left(-\gamma \sum_{h=j}^{k-1} \mathrm{E}_{h}^{(n)} / h\right)\right]
$$

Some algebraic manipulations yield

$$
\frac{T_{n}(f)}{y_{0}-Y_{n-k+1, n}}=f(k-1)-\left(\sum_{j=1}^{j=k-1}(f(j)-f(j-1)) \exp \left(-\gamma \sum_{h=j}^{k-1} \mathrm{E}_{h}^{(n)} / h\right)\right) .
$$


Set

$$
S_{j, k, n}=\exp \left(-\gamma \sum_{h=j}^{k-1} \mathrm{E}_{h}^{(n)} / h\right)
$$

and observe that for each $n \geq 1, S_{j, k, n}$ and $S_{j, k}$, which are defined in (7), have the same distribution. Let

$$
s_{j, k, n}=\mathrm{E}\left(S_{j, k, n}\right)=\exp \left(-\sum_{h=j}^{k-1} \log (1+\gamma / h)\right) .
$$

Then we have

$$
A_{k, n}(f)=f(k-1)-\sum_{j=1}^{k-1} \Delta f(j) s_{j, k, n}
$$

This yields

$$
W_{k-1, n}^{*}(f)=\sum_{j=1}^{k-1} \Delta f(j)\left(S_{j, k, n}-s_{j, k, n}\right) .
$$

Next, observe that for any $n \geq 1, W_{k(n)-1, n}^{*}(f)={ }_{d} W_{k(n)-1}$. Therefore, $W_{k(n)-1, n}^{*}(f)$ converges in distribution to $W_{\infty}(f)$ whenever $W_{k(n)-1}$ converges almost surely to $W_{\infty}(f)$, which completes the proof of the theorem.

\section{Application to extreme value theory}

\subsection{Asymptotic results in the Weibull case}

In this section, we are interested in the particular case of the Weibull distribution which obtained for $0<\tau<1 / 2$. For the general case, we have the following Karamata representation when $F$ is Weibull distributed with parameter $\gamma>0, x_{0}(F)<\infty$

$$
\log x_{0}-F^{-1}(1-u)=c(1+p(u)) u^{\gamma} \exp \left(\int_{u}^{1} b(t) t^{-1} d t\right)
$$


where $(p(u), b(u)) \rightarrow(0,0)$ as $u \rightarrow 0$. Theorem 2 dealt with the special case of $p(u)=b(u)=0$. In this section, we provide statistical tests for this special case and further consider perturbation models that arise with special cases of $b(u)$.

\subsection{Critical points of the d.f. of $W_{\infty}(f)$}

We use computer-based methods for approximating the law of $W_{\infty}(f)$. Simulation studies show that the empirical d.f. based of $B_{0}=1000$ replications are very stable from $k=2000$.

We proceed as follows. Fix $\tau$ in $0<\tau<1 / 2, \gamma>0$ and $k \geq 2000$. At each step, $B$ runs from 1 to $B_{0}=1000$. We generate standard exponential samples $E_{1}(B), \ldots, E_{k}(B)$ and compute $W_{k}^{*}$ denoted by $W_{k}^{*}(B)$. We finally consider the empirical d.f. denoted by $G_{k}$, based on $W_{k}^{*}(1), \ldots, W_{k}^{*}\left(B_{0}\right)$. Since $G_{k}$ is stable in the sense that it does not significantly change from $k=2000$, we approximate the d.f. $G_{\infty}$ of $W_{\infty}\left(f_{\tau}\right)$ by $G_{k}$ for large $k$. As an example, we give an illustration in Figure 1 the d.f. $G_{k}$ for $k$ in $\{250,500,750,1000,2000,5000\} ; \gamma=1$ and $\tau=1 / 4$. Here for instance, we infer that the support of $G_{\infty}$ is $[-0.5,0.5]$. Overall, the figures clearly establish stability and support our proposal. Users who are interested in using the method provided in this paper may require executable files from the authors, the computation of $P\left(W_{\infty}\left(f_{\tau}\right) \leq x\right)=G_{\infty}(x)$ and $P\left(\left|W_{\infty}\left(f_{\tau}\right)\right| \leq|x|\right)=$ $G_{\infty}(|x|)-G_{\infty}(-|x|)$ for $x \in \Re$.

Figure 1: Illustration of the distribution functions of $W_{k, n}(1 / 4)$ for different values of $\mathrm{k}$ 

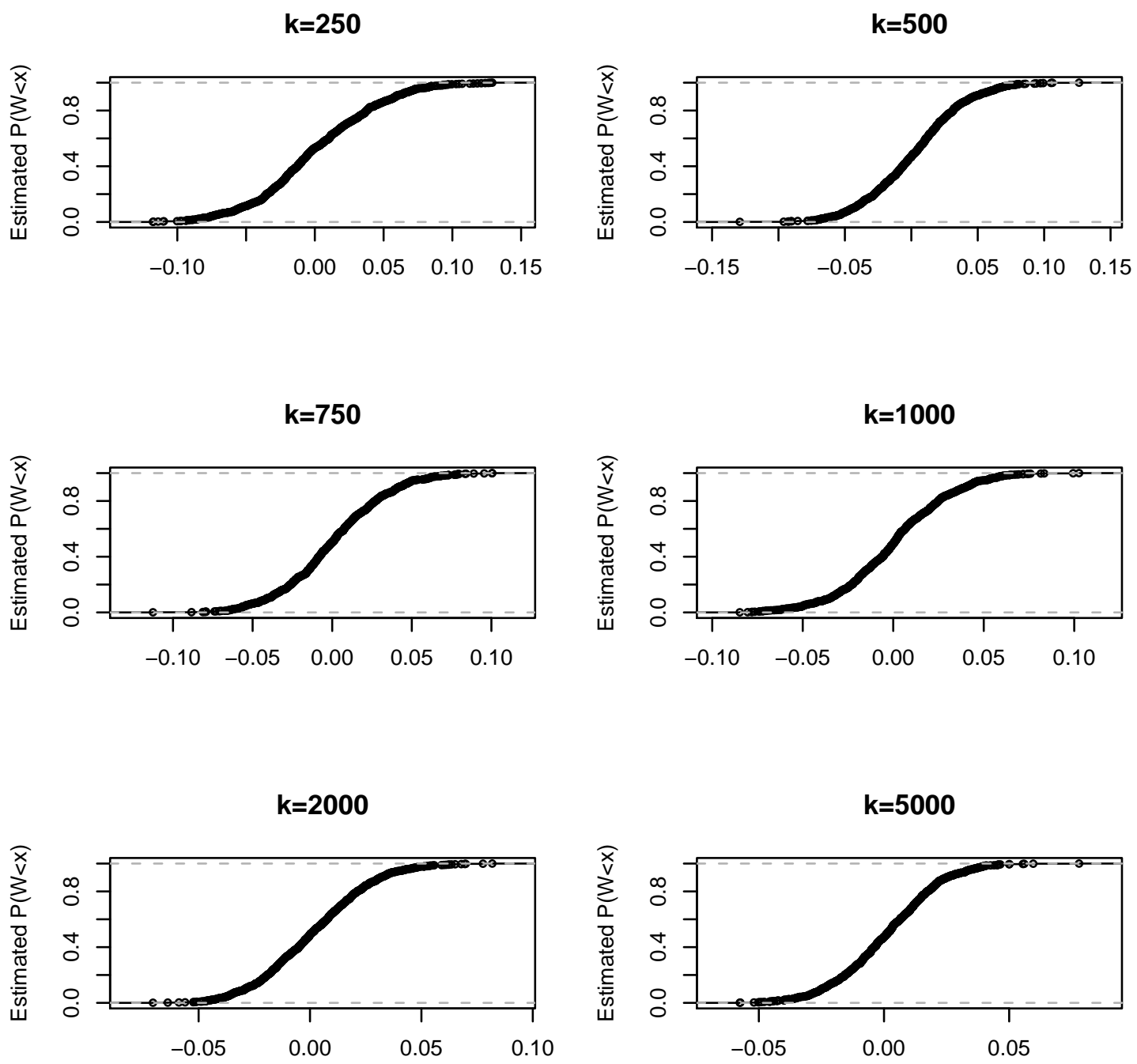


\begin{tabular}{llll}
\hline Models & Quantile functions & $T_{n}^{*}\left(f_{\tau}\right)$ & P-values \\
\hline Weibull 1 & $F^{-1}(1-u)=\exp \left(1-u^{\gamma}\right)$ & 3.16 & $67.4 \%$ \\
\hline Weibull 2 & $F^{-1}(1-u)=\exp \left(1-u^{\gamma}\left(1+u^{9}\right)\right)$ & 0.0367 & $38.9 \%$ \\
\hline Weibull 3 & $F^{-1}(1-u)=\exp \left(1-u^{\gamma}\left(1+u^{8}\right)\right)$ & 0.048 & $27.3 \%$ \\
\hline Weibull 4 & $F^{-1}(1-u)=\exp \left(1-u^{\gamma}\left(1+u^{7}\right)\right)$ & 3.063 & $13.2 \%$ \\
\hline Weibull 5 & $F^{-1}(1-u)=\exp \left(1-u^{\gamma}\left(1+u^{6}\right)\right)$ & 3.0725 & $10.4 \%$ \\
\hline Weibull 6 & $F^{-1}(1-u)=\exp \left(1-u^{\gamma}\left(1+u^{5}\right)\right)$ & 3.097 & $2 \%$ \\
\hline Weibull 7 & $F^{-1}(1-u)=\exp \left(1-u^{\gamma}\left(1+u^{4}\right)\right)$ & 3.17 & $0 \%$ \\
\hline Standard Exponential & $F^{-1}(1-u)=-\log u$ & 3.77 & $0 \%$ \\
\hline Pareto & $F^{-1}(1-u)=u^{-1}$ & 19.755 & $0 \%$ \\
\hline
\end{tabular}

Table 1: Statistical tests for four models using the law of $W_{\infty}(1 / 4)$

\subsection{Statistical tests}

In this subsection, we show how $G_{\infty}$ may be used to test the null hypothesis that $F \in D\left(G_{-\gamma}\right)$. The following approximation is used in the sequel.

$$
T_{n}^{*}(f)=T_{n}(f) /\left(y_{0}-\log X_{n-k+1, n}\right) \approx T_{n}(f) /\left(\log X_{n, n}-\log X_{n-k+1, n}\right) .
$$

For testing the null hypothesis that $F \in D\left(G_{-\gamma}\right)$, we compute the p-values for the seven models with $\gamma=1$, as described in Table 1. The first corresponds to the pure model for which $p(u)=b(u)=0, u \in(0,1)$. In the remaining six others, a shift of order $\left(1+u^{q}\right)$ is included in order to assess the impact of the perturbation, especially that of exponent $q$. We use $n=300$ and $k=200$. The conclusions that transpired are as follow: (i) the pure model is accepted with a large p-value around 68\%, (ii) the models Weibull 2 up to Weibull 6 , which correspond to shift parameters $q \in\{6,7,8,9\}$, are accepted with $p$-values at least greater than 10.4\%; and (iii) the Weibull 6 and Weibull 7 models, corresponding to shift parameters $q=4$ and $q=5$, and the exponential and Pareto models are rejected. This is reasonable since, as we pointed out earlier, the convergence depends on the functions $b$ and $p$ in (14) that are given by $p(u)=0$ and $b(u)=-q u^{q}\left(1+u^{q}\right)^{-1}$ and $c=1$. 


\section{Remarks and Discussion}

We observe that for the Weibull simple case, the law of the functional Hill process is found for $0<\tau<1 / 2$. For the general case, we have the following Karamata representation when $F$ is in the Weibull case of parameter $\gamma>0$ $: x_{0}(F)<\infty$ and

$$
\log x_{0}-F^{-1}(1-u)=c(1+p(u)) u^{\gamma} \exp \left(\int_{u}^{1} b(t) t^{-1} d t\right)
$$

where $(p(u), b(u)) \rightarrow(0,0)$ as $u \rightarrow 0$. In an upcoming paper we will study the dependence of the results on the auxiliary functions $p$ and $b$ and we also determine general conditions on $b$ and $p$ under which $T_{n}^{*}(f)=T_{n}(f) /\left(y_{0}-\right.$ $\left.\log X_{n-k+1, n}\right)$ behaves as $W_{k, n}^{*}$ as in the present case. Nevertheless, we will include in the statistical tests some models with specific forms of $b(\cdot)$ as shown in Table 1 and used in Subsection 4.3.

\section{Appendix}

This section is devoted to the computations of the moments of

$$
S_{j, k}=\exp \left(-\gamma \sum_{h=j}^{k-1} E_{h} / h\right)
$$

and to their approximations for large values of $j$, where the $E_{h}^{\prime} s$ are independent unit exponential r.v. We begin by giving a particular and useful tool for the expansion of the logarithm function. Let $\varepsilon>0$ be fixed. There exists $u_{0}>0$ such that

$$
0<u<u_{0}, \quad \log (1+u)=u+\theta(\varepsilon, u) u^{2}
$$

where $\theta(\varepsilon, u) \in[-\varepsilon-1 / 2, \varepsilon-1 / 2] \equiv A(\varepsilon)=\left[a_{1}(\varepsilon), a_{2}(\varepsilon)\right]$. For any integer 
$m \geq 1$, let $J_{0}(m)$ be a positive integer such that $J_{0}(m) \geq \gamma /\left(m u_{0}\right)$. Then, we have

$$
j \geq J_{0}(m) \Longrightarrow \log (1+\gamma / j)=\frac{j}{\gamma}+\theta_{j}\left(\frac{j}{\gamma}\right)^{2} \text { with } \theta_{j} \in A(\varepsilon) .
$$

This expansion 15 will be instrumental in the computations below. All coming numbers of the form $\theta_{j}$ and $\theta_{h}(r)$ stand as notations of the number $\theta(u, \varepsilon)$ in (15) depending of the form of $u$. In all cases, these numbers are in $A(\varepsilon)$.

\subsection{Moment estimation}

\subsubsection{Exact values}

For any integer $m \geq 1$, we have

$$
\begin{gathered}
E\left(S_{j, k}^{m}\right)=E \exp \left(-m \gamma \sum_{h=j}^{k-1} E_{h} / h\right)=\prod_{h=j}^{k-1} E \exp \left(-m \gamma E_{h} / h\right)=\prod_{h=j}^{k-1}(1+m \gamma / h)^{-1} \\
=\exp \left(-\sum_{h=j}^{k-1} \log (1+m \gamma / h)\right) .
\end{gathered}
$$

Now for $j \geq J_{0}(m)$,

$$
E\left(S_{j, k}^{m}\right)=\exp \left(-m \gamma \sum_{h=j}^{k-1}(1 / h)-m^{2} \gamma^{2} \sum_{h=j}^{k-1} \theta_{h} / h^{2}\right)
$$

Then for any $j$ and $k, \operatorname{Var}\left(S_{j, k}\right)$ is

$$
\exp \left(-2 \sum_{h=j}^{k-1} \log (1+2 \gamma / h)\right)-\exp \left(-2 \sum_{h=j}^{k-1} \log (1+\gamma / h)\right) \leq 1,
$$


since this is difference of two points in $[0,1]$. Later, we will need this notation,

$$
\begin{aligned}
E\left(S_{j, k}^{m}\right) & =\exp \left(-m \gamma \sum_{h=j}^{k-1}(1 / h)-m^{2} \gamma^{2} \sum_{h=j}^{k-1} \theta_{h} / h^{2}\right) \\
& =\exp \left(-m \gamma \sum_{h=j}^{k-1}(1 / h)\right) B(1, j, m)
\end{aligned}
$$

where

$$
B(1, j, m)=\exp \left(-m^{2} \gamma^{2} \sum_{h=j}^{k-1} \theta_{h} / h^{2}\right)
$$

\subsubsection{Approximate values for moments}

Combining Formula (16) above and Formula (34) in the last subsection 6.2 of this Appendix, leads to

$$
\begin{aligned}
\left|m^{2} \gamma^{2} \sum_{h=j}^{k-1} \theta_{h} / h^{2}\right| & \leq\left|a_{1}(\varepsilon)\right| m^{2} \gamma\left(\frac{1}{j}-\frac{1}{k-1}-\frac{1}{j^{2}}\right) \\
& \leq \frac{\left|a_{2}(\varepsilon)\right| m^{2} \gamma}{j}
\end{aligned}
$$

for $j \geq J_{0}(m)$.

Let $J_{1}(\varepsilon, m)$ be a positive integer such that $\frac{\left|a_{1}(\varepsilon)\right| m^{2} \gamma}{J_{1}(\varepsilon, m)} \leq \varepsilon$. Then we have

$$
j \geq J_{1}(\varepsilon, m) \vee J_{0}(m) \Longrightarrow \exp \left(-m^{2} \gamma^{2} \sum_{h=j}^{k-1} \theta_{h} / h^{2}\right) \leq e^{\varepsilon}
$$

From the definition of $B(1, j, m)$ in 20 above and from 21 , we have for $j \geq J_{0}(m)$ 


$$
B(1, m, j)=1+O\left(\frac{\left|a_{1}(\varepsilon)\right| m^{2} \gamma}{j}\right)=1+O\left(j^{-1}\right)
$$

for $m$ fixed, since $\gamma$ is fixed in all the text. Next, by denoting

$$
B(2, m, j)=\exp \left(-m \gamma\left\{\sum_{h=j}^{k-1} \frac{1}{h}-\log ((k-1) / j)\right\}\right)
$$

we get by 33 that

$$
\exp (-(m \gamma) / j) \leq B(1, m, j) \leq \exp (-(m \gamma) /(k-1))
$$

so that, since $1 \leq j \leq k$ and since $\gamma$ is fixed, for $m$ fixed,

$$
B(1, m, j)=1+O\left(j^{-1}\right), \quad \text { as } \mathrm{j} \text { increases indefinitely. }
$$

Now, since

$$
\exp \left(-m \gamma \sum_{h=j}^{k-1}(1 / h)\right)=\left(\frac{j}{k-1}\right)^{m \gamma} \exp \left(-m \gamma\left\{\sum_{h=j}^{k-1} \frac{1}{h}-\log ((k-1) / j)\right\}\right)
$$

we have, from Formula (18) in the last subsection of this appendix and Formula $(22)$ above, that

$$
E\left(S_{j, k}^{m}\right)=\left(\frac{j}{k-1}\right)^{m \gamma} B(1, m, j) B(2, m, j),
$$

with, for $m$ fixed and for $j \geq J_{1}(\varepsilon, m) \vee J_{0}(m)$,

$$
B(1, m, j)=1+O\left(j^{-1}\right) \text { and } B(2, m, j)=1+O\left(j^{-1}\right) .
$$




\subsubsection{Approximate values for variances}

We have for $j>J_{0}(2)$

$$
E\left(S_{j, k}^{2}\right)=\exp \left(-2 \gamma \sum_{h=j}^{k-1}(1 / h)-4^{2} \gamma^{2} \sum_{h=j}^{k-1} \theta_{h}(1) / h^{2}\right) .
$$

and for $j>J_{0}(1)$

$$
\begin{gathered}
E\left(S_{j, k}\right)^{2}=\exp \left(\left(-\gamma \sum_{h=j}^{k-1}(1 / h)-\gamma^{2} \sum_{h=j}^{k-1} \theta_{h}(2) / h^{2}\right)\right)^{2} \\
=\exp \left(-2 \gamma \sum_{h=j}^{k-1}(1 / h)-2 \gamma^{2} \sum_{h=j}^{k-1} \theta_{h}(2) / h^{2}\right) .
\end{gathered}
$$

Thus

$$
\operatorname{Var}\left(S_{j}^{*}\right)=\exp \left(2 \gamma \sum_{h=j}^{k-1} 1 / h\right) \times V(2,2, j)
$$

where

$V(2,2, j)=\left\{\exp \left(-4^{2} \gamma^{2} \sum_{h=j}^{k-1} \theta_{h}(1) / h^{2}\right)-\exp \left(-2 \gamma^{2} \sum_{h=j}^{k-1}\left(2 \theta_{h}(1)-\theta_{h}(2)\right) / h^{2}\right)\right\}$.

Now, the same technique used in Formula (23) above proves that for $m=2$,

$$
\exp \left(2 \gamma \sum_{h=j}^{k-1} 1 / h\right)=\left(\frac{j}{k-1}\right)^{2 \gamma} V(1,2, j),
$$

with $V(1,2, j)=1+O\left(j^{-1}\right)$ as $j \rightarrow+\infty$.

Let us now handle $V(2,2, j)$. Since $x=4^{2} \gamma^{2} \sum_{h=j}^{k-1} \theta_{h}(1) / h^{2}$ and $y=2 \gamma^{2} \sum_{h=j}^{k-1} \theta_{h}(2) / h^{2}$ 
are both nonnegative, we have $\left|e^{-x}-e^{-y}\right| \leq|x-y|$. Thus

$$
\begin{aligned}
0 & \left.\leq \exp \left(-4^{2} \gamma^{2} \sum_{h=j}^{k-1} \theta_{h}(1) / h^{2}\right)-\exp \left(-2 \gamma^{2} \sum_{h=j}^{k-1} \theta_{h}(2)\right) / h^{2}\right) \\
& \leq 2 \gamma^{2} \sum_{h=j}^{k-1}\left|2 \theta_{h}(1)-\theta_{h}(2)\right| / h^{2} \leq \frac{2 \gamma^{2}\left|a_{2}(\varepsilon)\right|}{j},
\end{aligned}
$$

by (34). Therefore

$$
\operatorname{Var}\left(S_{j, k}\right)=\left(\frac{j}{k-1}\right)^{2 \gamma} V(1,2, j) V(2,2, j)
$$

with

$$
|V(1,2, j)|=1+O\left(j^{-1}\right) \text { and } V(2,2, j)=1+O\left(j^{-1}\right) .
$$

\subsubsection{Covariance approximate values}

Let $\ell>1$ and consider $\sigma_{j, j+\ell}=\operatorname{cov}\left(S_{j+\ell, k}, S_{j, k}\right)$. We have

$$
\begin{aligned}
E\left(S_{j, k}\right) & =\exp \left(\sum_{h=j}^{k-1}-\log (1+\gamma / h)\right) \\
& =\exp \left(\sum_{h=j}^{j+\ell-1}-\log (1+\gamma / h)\right) \exp \left(\sum_{h=j+l}^{k-1}-\log (1+\gamma / h)\right) \\
& =E\left(S_{j+\ell, k}\right) \exp \left(\sum_{h=j}^{j+\ell-1}-\log (1+\gamma / h)\right)
\end{aligned}
$$


Further,

$$
\begin{aligned}
S_{j, k} S_{j+\ell, k} & =\exp \left(-\gamma \sum_{h=j}^{k-1} E_{h} / h\right) \exp \left(-\gamma \sum_{h=j+\ell}^{k-1} E_{h} / h\right) \\
& =\exp \left(-\gamma \sum_{h=j}^{j+\ell-1} E_{h} / h-\gamma \sum_{h=j+\ell}^{k-1} E_{h} / h\right) \exp \left(-\gamma \sum_{h=j+\ell}^{k-1} E_{h} / h\right) \\
& =\exp \left(-2 \gamma \sum_{h=j+\ell}^{k-1} E_{h} / h\right) \exp \left(-\gamma \sum_{h=j}^{j+\ell-1} E_{h} / h\right) \\
& =S_{j+\ell, k}^{2} \exp \left(-\gamma \sum_{h=j}^{j+\ell-1} E_{h} / h\right)
\end{aligned}
$$

Hence

$$
E\left(S_{j, k} S_{j+\ell, k}\right)=E\left(S_{j+\ell, k}\right)^{2} \exp \left(\sum_{h=j}^{j+\ell-1}-\log (1+\gamma / h)\right) .
$$

For $j \geq J_{0}(1) \vee J_{0}(2)$,

$$
\begin{aligned}
\operatorname{cov}\left(S_{j, k} S_{j+\ell, k}\right) & =\operatorname{Var}\left(S_{j+\ell, k}\right) \exp \left(\sum_{h=j}^{j+\ell-1}-\log (1+\gamma / h)\right) \\
& =\operatorname{Var}\left(S_{j+\ell, k}\right) \exp \left(-\gamma \sum_{h=j}^{j+\ell-1} 1 / h-\gamma^{2} \sum_{h=j}^{j+\ell-1} \theta_{h} / h^{2}\right) \\
& =\operatorname{Var}\left(S_{j+\ell, k}\right)\left(\frac{j}{j+\ell-1}\right)^{\gamma}\left(1+O\left(j^{-1}\right)\right)
\end{aligned}
$$

\subsection{Integrals computations}

Let $b \geq 1$. By comparing the area under the curve of $f(x)=x^{-b}$ going from $j$ to $k-1$ and that of the rectangles based on the intervals $[h, h+1]$, 
$h=1, . ., k-2$, we obtain

$$
\sum_{h=j+1}^{k-1} h^{-b} \leq \int_{j}^{k-1} x^{-b} d x \leq \sum_{h=j}^{k-2} h^{-b}
$$

that is

$$
\int_{j}^{k-1} x^{-b} d x+(k-1)^{-b} \leq \sum_{h=j}^{k-1} h^{-b} \leq \int_{j}^{k-1} x^{-b} d x+j^{-b} .
$$

Likely, comparing the area under the curve of $f(x)=x^{b}$ going from $j$ to $k-1$ and those of the rectangles based on the intervals $[h, h+1], j=1, . ., k-2$, we also get

$$
\int_{j}^{k-1} x^{b} d x+j^{b} \leq \sum_{h=j}^{k-1} h^{b} \leq \int_{j}^{k-1} x^{b} d x+(k-1)^{b} .
$$

Next, for $b=1$ and $b=2$, (31) yields

$$
\frac{1}{j} \leq \log ((k-1) / j)-\sum_{h=j}^{k-1} \frac{1}{h} \leq \frac{1}{k-1} .
$$

and

$$
\frac{1}{j}-\frac{1}{k-1}-\frac{1}{j^{2}} \geq \sum_{h=j}^{k-1} h^{-2} \geq \frac{1}{j}-\frac{1}{k-1}-\frac{1}{(k-1)^{2}},
$$

respectively. Combining both implications, we further get

$$
\frac{1}{j^{2}} \leq \frac{1}{j}\left(1-\frac{j}{k-1}\right)-\sum_{h=j}^{k-1} h^{-2} \leq \frac{1}{(k-1)^{2}} .
$$

Acknowledgement 1 The authors thank the unknown referee whose detailed and careful reading, and comments and suggestions significantly helped in making the paper better in all its aspects. 
Acknowledgement 2 The two first authors acknowledge support from the World Bank Excellence Center (CEA-MITIC) of Saint-Louis, Senegal, that is continuously funding their research activities starting 2014.

\section{References}

[1] Beirlant, J. Goegebeur, Y. and Teugels, J.(2004). Statistics of Extremes Theory and Applications. Wiley. (MR2108013)

[2] Csörgő, S., Deheuvels, P. and Mason, D. M.(1985). Kernel estimates of the tail index of a distribution. Ann. Statist., 13, 1050-1077. (MR0803758)

[3] Daley, D.J.(1968). Stochastically monotone Markov chains, Z. Wahrsch. theor. verw Gebiete, 10,305-317.

[4] Dème E., Lo G.S. and Diop, A.(2012). On the generalized Hill process for small parameters and applications. Journal of Statistical Theory and Application, 11(2), pp. 397-418. Doi : 10.2991/jsta.2013.12.1.3

[5] Lo G.S., Dème E.(2012). A Functional Generalized Hill Process and Its Uniform Theory. International Journal of Statistics and Probability, 1(2), pp. 250-268. Doi:10.5539/ijsp.v1n2p250

[6] Diop, A. and Lo, G.S.(1994). Sur une caractérisation statistique simple des extrêmes. Afrika Mat., Série 3, Vol. (3), 81-95. (Zbl 0819.60052) (MR1430638)

[7] Diop, A. and Lo G. S.(2009). Ratio of generalized Hill's estimator and its asymptotic normality theory. Math. Method. Statist., 18(2), pp. 117-133. (MR2537361) 
[8] Embrechts, P., Küppelberg C. and Mikosh T. (1997). Modelling extremal events for insurance and Finance. Springer Verlag.

[9] Galambos, J.(1985). The Asymptotic Theory of Extreme Order Statistics. Wiley, New-York. (MR0489334)

[10] Goegebeur, Y., Beirlant, J. and de Wet, T.(2010). Kernel estimators for the second order parameter in extreme value statistics. J. Statist. Plann. Inference, 140 (9), 2632-2652. (MR2644084)

[11] Groeneboom, Lopuhaä, H.P and Wolf, P.P.(2003). Kernel-type estimator for the extreme values index. Ann. Statist., 31, (6), pp. 1956-1995.

[12] de Haan, L. and Ferreira A. (2006). Extreme Value Theory: An Introduction. Springer. (MR2234156)

[13] Karlin, S. and Howard M.,Taylor (1975). A First Course in Stochastic Processes. Academic Press.

[14] Lo, G.S.(2016). Weak Convergence (IA). Sequences of random vectors. SPAS Books Series.(2016). Doi : 10.16929/sbs/2016.0001. Arxiv : 1610.05415

[15] Loève, M.(1977). Probability Theory I. Springer-Verlag. New-York.

[16] Resnick, S.I.(1987). Extreme Values, Regular Variation and Point Processes. Springer-Verbag, New-York.

[17] Shorack G.R. and Wellner J. A.(1986). Empirical Processes with Applications to Statistics. Wiley-Interscience, New-York. (MR0838963) 